\title{
Fractional differential equation with uncertainty
}

\author{
Karanveer Singh, R N Prajapati \\ Department of Mathematics \\ University Institute of Sciences \\ Chandigarh University, Gharuan \\ Mohali,Punjab-140413,India \\ karanveersinghkvskkt@gmail.com \\ narain.prajapati@gmail.com
}

\begin{abstract}
We consider a fractional order differential equation with uncertainty and introduce the concept of solution. It goes beyond ordinary first-order differential equations and differential equations with uncertainty.

Keywords:- Fractional differential equation, Differential equation with uncertainty.
\end{abstract}

\section{Introduction}

Fractional calculus is a generalization of ordinary differentiation and integration to an arbitrary non-integer order. The subject is as old as the differential calculus and dates back to the time when Leibnitz and Newton invented the differential calculus. The idea of fractional calculus has been a topic of interest not only among mathematicians, but also among physicists and engineers. Fractional calculus appears in electrochemistry, viscoelasticity, electromagnetism, rheology, etc. For more details, see the monographs by Kiryakova [1], Lakshmikantham and Vatsala [2], Kilbas et al.[3]. Miller and Ross [4], and the references therein. Some authors have considered initial value problems for fractional differential equations. Some contributions to the theory can be seen in $[5,6,7,8,9,10]$. To study some dynamic processes it is necessary to take into account imprecision, randomness or uncertainty. The uncertainty can be modeled by incorporating it into the dynamic system and considering fuzzy differential equations.

Let $\alpha \in(0,1], \mathrm{T}>0$ and $\mathrm{E}$ be the set of fuzzy real numbers.[11, 12]

In this paper we consider a differential equation with uncertainity of the type

$$
D^{\alpha} x(t)=f(t, x(t)), t \in(0, T]
$$


where $\mathrm{f}[0, \mathrm{~T}] \times E \rightarrow E$ is continuous. We will consider this equation with some adequate initial condition for a given $x_{0} \in \mathrm{E}$.

If $f[0, T] \times R \rightarrow R$, the (1) reduces to a fractional differential equation.

If $\alpha=1$, then (1) is just a first order fuzzy differential equation.

Here we combine both types of uncertainty differential equations and fractional order to consider a new type of dynamic system: fractional order fuzzy differential equations. Then we present the concept of solution for these types of equations.

\section{Fractional differential equations}

Fractional differential equations (FDEs) have fractional derivatives of the type $\left.\left(d^{\alpha}\right) / d x^{\alpha}\right)$ that are defined for $\alpha>0$, where $\alpha$ is not always an integer.

Let $\alpha \in(0,1], T>0$, and $\sigma:(0, T] \rightarrow \mathrm{R}$.

Consider the

differential

$$
\begin{gathered}
D^{\alpha} x(t)=f(t, x(t)), t \in(0, T] . \\
D^{\alpha} x(t)=\frac{1}{\Gamma(1-\alpha)} \frac{d}{d t} \int_{0}^{t}(t-s)^{-\alpha} x(s) d s
\end{gathered}
$$

nonlinear

equation

where

is the usual Reimann-Liouville fractional derivative of order $\alpha$ of the function $x$ : $(0, T] \rightarrow \operatorname{Rand} f[0, T] \times R \rightarrow R$ is continuous.

The initial condition in this case

$$
\lim _{t \rightarrow 0^{+}} t^{1-\alpha} x(t)=x_{0} \in \mathbb{R}
$$

For $\alpha \in \mathrm{R}$ the corresponding linear equation is

$$
D^{\alpha} x(t)+a x(t)=\sigma(t), t \in J:=(0, T] .
$$

Consider the linear fractional differential equation (4) with the initial condition

(3). The solution is given by [1]:

$$
x(t)=x_{0} \Gamma(\alpha) t^{\alpha-1} E_{\alpha, \alpha}\left(-a t^{\alpha}\right)+\int_{0}^{t}(t-s)^{\alpha-1} E_{\alpha, \alpha}\left(-a(t-s)^{\alpha}\right) \sigma(s) d s,
$$

where $E_{\alpha, \alpha}$ is the classical Mittag-Leffler function 


$$
E_{\alpha, \alpha}(z)=\sum_{k=0}^{\infty} \frac{z^{k}}{\Gamma(\alpha(k+1))}
$$

We point out that for $\alpha=1$ this representation is still valid since $\Gamma(1)=1$ and $E_{1,1}(z)=e^{z}$. We thus obtain the solution

$$
x(t)=x_{0} e^{-a t}+\int_{0}^{t} e^{-a(t-s)} \sigma(s) d s
$$

of the initial problem for a linear first order ordinary differential equation

$$
x^{0}(t)+a u(t)=\sigma(t), x(0)=x_{0}
$$

\section{Differential equations with uncertainty}

Let $E$ be the set of fuzzy real numbers [13] and consider the nonlinear differential equation with uncertainty

$$
x^{0}(t)=f(t, x(t)), t \in(0, T],
$$

where $f[0, T] \times E \rightarrow E$ is continuous , and initial condition

$$
x(0)=x_{0} \in E .
$$

For $a>0$ the solution of the linear problem

$$
x^{0}(t)=a x(t)+\sigma(t), t \in(0, T]
$$

with the initial condition (7) is given by [14]

$$
x(t)=e^{a t}\left(x_{0}+\int_{0}^{t} e^{-a s} \sigma s d s\right) .
$$

\section{Fractional differential equations with uncertainty}

We now consider the fractional equation with uncertainty(1),i.e.,

$$
D^{\alpha} x(t)=f(t, x(t)), t \in(0, T],
$$

Where $f:[0, T] \times E \rightarrow E$ is continuous.

For a given $x_{0} \in E$ the appropriate initial condition is

$$
\lim _{t \rightarrow 0^{+}} t^{1-\alpha} x(t)=x_{0} \in E .
$$

Assume that there exists $\lambda>0$ such that the nonlinearity $\mathrm{f}$ is of the form

$$
f(t, x)=\lambda x+g(t, x)
$$


with $g:[0, T] \times E \rightarrow E$ continuous.

It is important to note that for a crisp differential equation, of integer or fractional order, the problem

$$
D^{\alpha} x(t)+a x(t)=\sigma(t), t \in(0, T]
$$

is the same as

$$
D^{\alpha} x(t)=-a x(t)+\sigma(t), t \in(0, T]
$$

However in the case of space $E$ both problems are not equivalent.

We write the Eq.(1) as

$$
D^{\alpha} x(t)=\lambda x(t)+g(t, x(t)), t \in(0, T]
$$

Hence we define a solution of this Eq.(10) as a function $x:(0, T] \rightarrow E$ such that

$$
x(t)=\Gamma(\alpha) t^{\alpha-1} E_{\alpha, \alpha}\left(\lambda t^{\alpha}\right) x_{0}+\int_{0}^{t}(t-s)^{\alpha-1} E_{\alpha, a l p h a}\left(\lambda(t-s)^{\alpha}\right) g(s, x(s)) d s .
$$

Moreover this solution satisfies the initial condition (9),i.e., $\lim _{t \rightarrow 0^{+}} X(t)=x_{0}$. Note that formally this expression is the same as the solution given by (5) with $\lambda=-a$.

\section{References}

[1] A. McBride, "V. kiryakova generalized fractional calculus and applications (pitman research notes in mathematics vol. 301, longman1994), 388 pp., 0 58221977 9,f 39.," Proceedings of the Edinburgh Mathematical Society, vol. 38, no. 1, pp. 189-190, 1995.

[2] V. Lakshmikantham and A. S. Vatsala, "Basic theory of fractional differential equations," Nonlinear Analysis: Theory, Methods \& Applications, vol. 69, no. 8, pp. 2677-2682, 2008.

[3] A. Kilbas, H. Srivastava, and J. Trujillo, Theory and Applications of Fractional Differential Equations. Amsterdam: Elsevier Science B.V, 2006.

[4] K. S. Miller and B. Ross, An introduction to the fractional calculus and fractional differential equations. Wiley, 1993.

[5] B. Ahmad and J. J. Nieto, "Existence results for a coupled system of nonlinear fractional differential equations with three-point boundary conditions," Computers \& Mathematics with Applications, vol. 58, no. 9, pp. 1838-1843, 2009. 
[6] D. Araya and C. Lizama, "Almost automorphic mild solutions to fractional differential equations," Nonlinear Analysis: Theory, Methods \& Applications, vol. 69, no. 11, pp. 3692-3705, 2008.

[7] B. Bonilla, M. Rivero, L. Rodr'ı́guez-Germ'a, and J. J. Trujillo, "Fractional differential equations as alternative models to nonlinear differential equations," Applied Mathematics and computation, vol. 187, no. 1, pp. 7988, 2007.

[8] Y.-K. Chang and J. J. Nieto, "Some new existence results for fractional differential inclusions with boundary conditions," Mathematical and Computer Modelling, vol. 49, no. 3-4, pp. 605-609, 2009.

[9] K. Diethelm and N. J. Ford, "Analysis of fractional differential equations," Journal of Mathematical Analysis and Applications, vol. 265, no. 2, pp. 229248, 2002.

[10] S. Zhang, "Monotone iterative method for initial value problem involving riemann-liouville fractional derivatives," Nonlinear Analysis: Theory, Methods \& Applications, vol. 71, no. 5-6, pp. 2087-2093, 2009.

[11] J. J. Nieto, R. Rodriguez-Lopez, and D. Franco, "Linear first-order fuzzy differential equations," International Journal of Uncertainty, Fuzziness and Knowledge-Based Systems, vol. 14, no. 06, pp. 687-709, 2006.

[12] J. J. Nieto, R. Opez, and D. Georgiou, "Fuzzy differential systems under generalized metric spaces approach," Dynamic Systems and Applications, vol. 17, no. 1, p. 1, 2008.

[13] P. Diamond and P. Kloeden, Metric spaces of fuzzy sets: theory and applications. World scientific, 1994.

[14] V. Lakshmikantham and R. N. Mohapatra, Theory of fuzzy differential equations and inclusions. CRC press, 2004. 